\title{
Packing sulfur into carbon framework for high volumetric performance lithium-sulfur batteries
}

\author{
Chen Zhang ${ }^{1,2}$ and Quan-Hong Yang ${ }^{1,2,3^{*}}$
}

Low volumetric performance is a common bottleneck of carbon-based electrode materials for practical applications, owing to the low density of porous carbons caused by the intrinsic void space [1]. Specifically, lithium-sulfur (Li-S) batteries as a hot topic of next-generation energy storage devices face the same dilemma that we have to balance their intrinsically electrochemical performance and volumetric performance [2-4]. The use of conductive porous carbon materials in the cathode of Li-S batteries, such as mesoporous carbon, carbon nanotube and graphene-derived carbons, can effectively accelerate the reaction kinetics, improve the electrochemical performance of sulfur cathode and promote the practical application of insulting sulfur $[3,5]$. However, addition of these materials results in massive void space in the electrode, which cannot meet the requirement of compact structure in real applications [6]. Such electrodes usually deliver relatively low volumetric performance even though their gravimetric performance can reach a high value.

The significance of volumetric performance for electrochemical energy storage (EES) devices has been addressed by our group previously, especially for carbon-based electrodes in supercapacitors, Li-S batteries and so on [1]. It is noted that a very effective approach for improving the volumetric performance of carbon-based electrodes and EES devices is densifying the electrode materials. The density change of carbon-based electrode materials thus will alter the microstructure. In Li-S batteries, the cathode consists of porous carbon and sulfur. Generally, the density of porous carbon is in a very low level $\left(0.3-0.6 \mathrm{~g} \mathrm{~cm}^{-3}\right)[7,8]$, while the density of bulk sulfur is above $2.0 \mathrm{~g} \mathrm{~cm}^{-3}$ [9]. Thus, the integration of carbon and sulfur is actually the combination of a low-density component and a high-density one. The precondition is that the density of sulfur is not changeable while the density of porous carbon materials can be tuned by controlling their microstructure, such as the pore volume, surface area, etc. [8,10-12]. It is of great interest that a density ceiling of porous carbon materials is of about $2.2 \mathrm{~g} \mathrm{~cm}^{-3}$, which is the density of graphite with the most compact structure. Thus, sulfur is always the heavier phase in carbon/sulfur cathode, while carbon is always the lighter one.

Based on the understanding of the role of carbon and sulfur in the hybrid electrode, the remedy for improving the density of carbon/sulfur cathode can be classified into two approaches as shown in Fig. 1: improving the sulfur fraction and densifying the carbon matrix. Zhang et al. [13] pioneered the research on the volumetric performance of carbon/sulfur cathode. They fabricated a series of carbon nanotube (CNT)/sulfur hybrids with different sulfur contents to tailor the evolution of the density of the hybrids and their corresponding volumetric performance. A high sulfur fraction up to $90 \mathrm{wt}$.\% was realized with a density of $1.98 \mathrm{~g} \mathrm{~cm}^{-3}$ for the hybrid material, and it shows an ultrahigh volumetric capacity and as well as a high volumetric energy density for the test cell (Fig. 2). A trick for this study is the carbon matrix they used, which has a unique conductive network and unimpeded space for sulfur impregnating. High sulfur fraction leads to a more insulting property due to the dominating insulting sulfur, and thus, the selection of carbon matrix is of extreme significance to tolerate and accommodate the high fraction of sulfur. The aligned CNT arrays provide enough space for storing a large amount of sulfur and the vertically aligned CNTs construct a spatially conductive network. Sulfur can readily diffuse into the inner space of the CNT arrays in contrast to that most of sulfur will be blocked on the external surface if a microporous carbon is employed. The volumetric capacity for electrode and volumetric energy density for the test cell are both evaluated, pioneering the promising topic for the development of Li-S batteries. This work not just inspires us how to improve the volumetric performance of

\footnotetext{
${ }^{1}$ Key Laboratory for Green Chemical Technology of Ministry of Education, School of Chemical Engineering and Technology, Tianjin University, Tianjin 300072, China

${ }^{2}$ Collaborative Innovation Center of Chemical Science and Engineering, Tianjin 300072, China

${ }^{3}$ Shenzhen Key Laboratory for Graphene-based Materials and Engineering Laboratory for Functionalized Carbon Materials, Graduate School at Shenzhen, Tsinghua University, Shenzhen 518055, China

*Corresponding author (email: qhyangcn@tju.edu.cn or yang.quanhong@sz.tsinghua.edu.cn)
} 


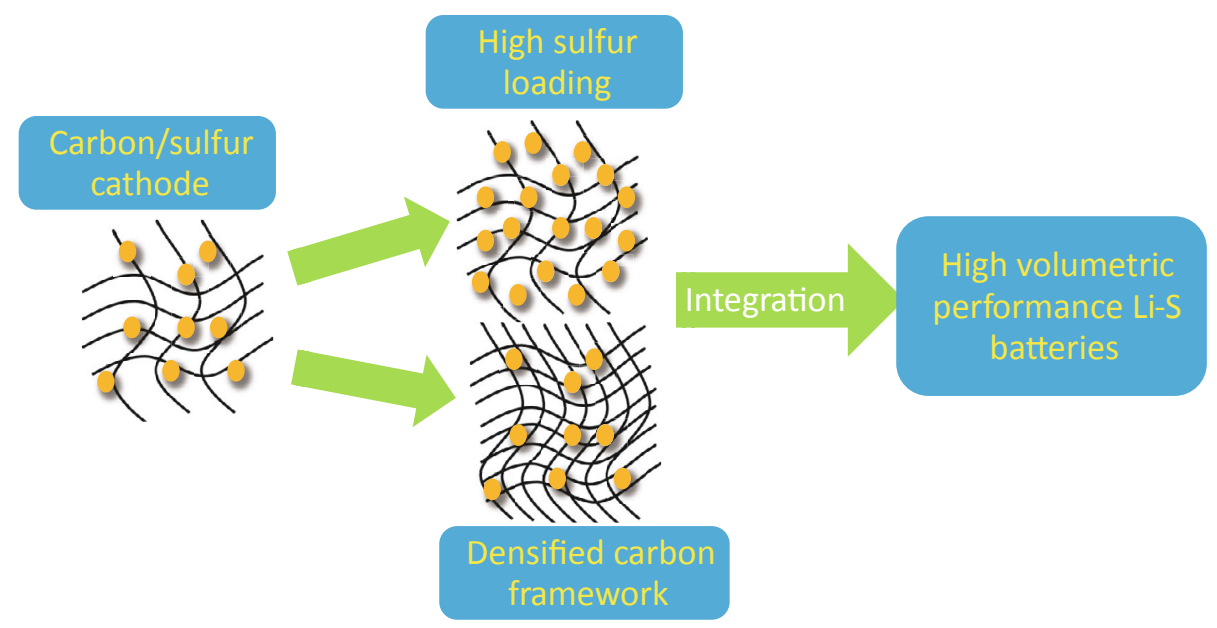

Figure 1 Illustration of the solutions to realize the fabrication of high volumetric performance Li-S batteries.

a
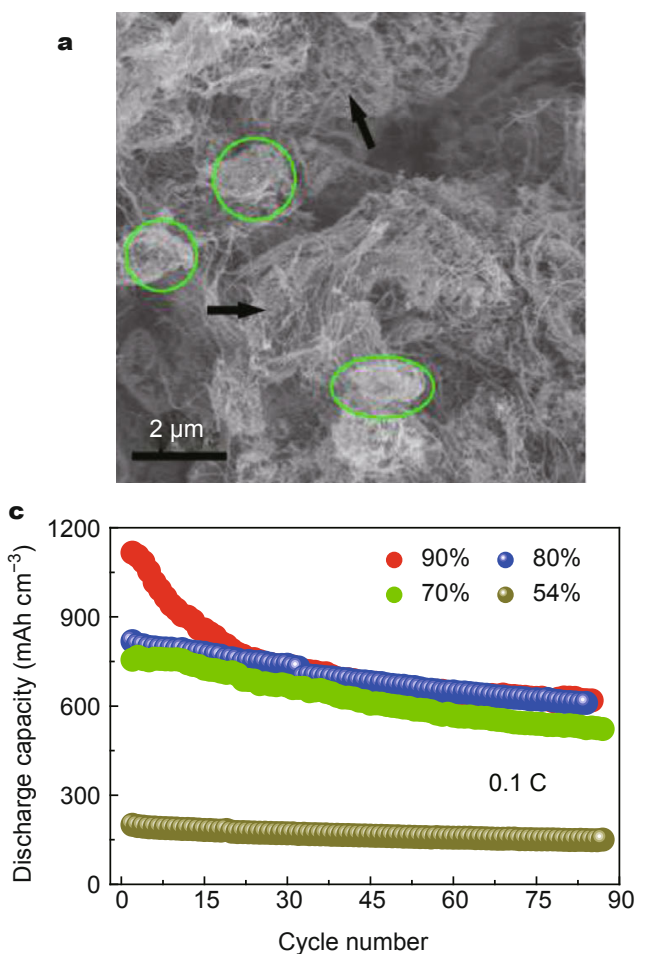

b
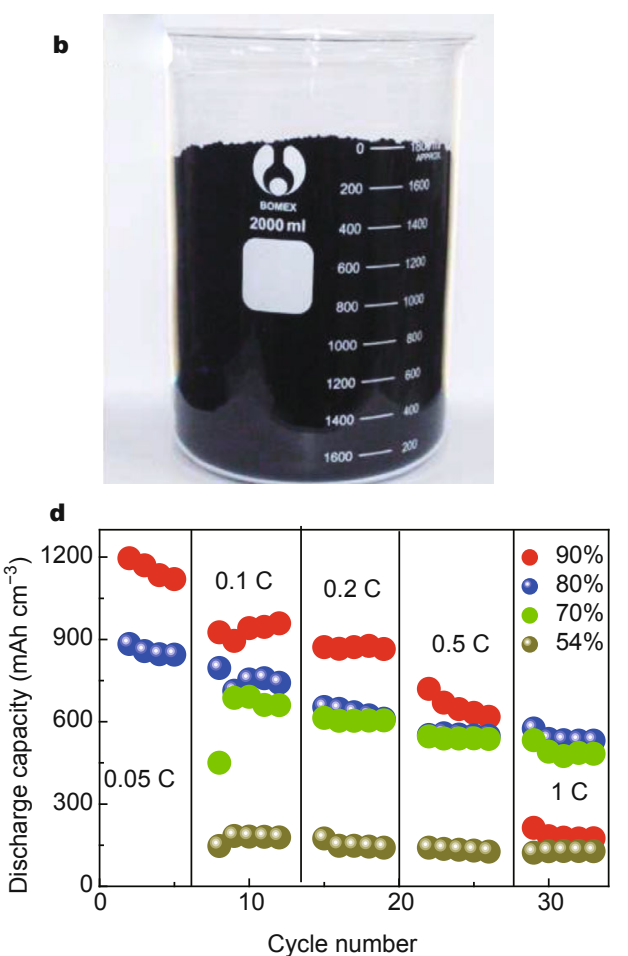

Figure 2 (a) SEM image and (b) photograph of the CNT/sulfur hybrid. Circles and arrows in (a) refer to the bulk sulfur particles and aligned CNTs. (c and d) Cycle performance and rate capability of CNT/sulfur cathode with different sulfur content. Images were reproduced with permission from Ref. [13], Copyright 2014, Elsevier.

Li-S batteries, and more importantly, it paves the avenue towards the industrial scale production of carbon/sulfur electrode and high performance Li-S batteries [14-19]. It can also be indicated that a spatial 3D carbon structure is more responsible for a high sulfur loading, and several studies have demonstrated that the $3 \mathrm{D}$ structure with high sulfur loading can bring high area capacity as well as potentially high volumetric performance [20-22].

To maintain high sulfur fraction and excellent electrochemical performance, the design and fabrication of carbon matrix with suitable structure is still challenging. Otherwise, high sulfur fraction usually leads to inhomo- 
geneous loading and poor electrical conductivity of the cathode. Being of equal significance, densifying the carbon matrix is another route to improve the volumetric performance of carbon/sulfur electrode. Compared with improving the sulfur fraction in a well-designed carbon matrix, densifying the carbon matrix focuses more on the materials design and texture control. More importantly, the dense structure is favorable for the confinement of sulfur and polysulfides. The main barrier for this approach is how to realize the control of the density of carbon matrix and how to guarantee the effective contact between carbon and sulfur. Our group previously realized the fabrication of highly dense but porous graphene assembly with high volumetric capacitance as electrode for supercapacitors. What drives the densifying of the graphene assembly is the capillary force between the graphene sheets and water during the evaporation of water in the graphene hydrogel $[8,23]$. In this process, the nondestructively drying process simultaneously brings the highly dense carbon and inherited porous structure, which are ideal characterizations for the carbon matrix of cathode materials in Li-S batteries with high volumetric performance.

Zhang et al. [24] proposed an effective solution for the fabrication of high density graphene/sulfur cathode. Similar to our previous study, they employed the freeze-drying or evaporation-induced drying to prepare two graphene assemblies with totally different densities and pore structures. To investigate the volumetric performance of the cathode using these two carbon matrixes, sulfur was loaded by a soft approach on graphene sheets and different drying processes were used to tune the microstructure of these hybrids. The compact graphene/sulfur hybrid could better facilitate the interaction between graphene and sulfur, while a more closed pore structure could constrain the sulfur and polysulfide. The dense graphene/sulfur hybrid shows much better cycle and rate performance, as well as a much higher volumetric capacitance, than the loose sample.

Our group also made some advances on the graphene/ sulfur cathode with high volumetric performance [25]. Other than the post-impregnating of sulfur into the graphene hydrogel by Zhang et al. [26], we realized the pre-impregnating of sulfur and the simultaneous formation of graphene hydrogel by $\mathrm{H}_{2} \mathrm{~S}$ inspired by our previous work. Pre-impregnating sulfur in graphene oxide (GO) solution can guarantee the homogeneous contact between the GO sheets and sulfur, which is critical for the corresponding electrochemical performance of the hybrid electrode as shown in Fig. 3a. Two essential processes are responsible for the formation of high-density graphene/ sulfur (HDGS). First, the partial reduction of the GO by $\mathrm{H}_{2} \mathrm{~S}$ triggers the spatial assembly of reduced graphene oxide (rGO) due to the amphiphilic nature of the rGO sheets
[27-29]. Second, the evaporation-induced drying process facilitates the driving force for the shrinkage of graphene/ sulfur hydrogel. As a result, a graphene/sulfur hybrid with high density, up to $1.53 \mathrm{~g} \mathrm{~cm}^{-3}$ and with robust structure (Fig. 3b), was fabricated. More interestingly, owing to the pre-loaded sulfur on the graphene sheets, the as-prepared sample is characterized by abundant ink bottle-like pores, whose structure is similar to the "Shumai dumplings" with graphene skin and wrapped sulfur stuff and this structure is beneficial for the confinement of sulfur as shown in Fig. 3c. Accordingly, HDGS with unique pore structure delivers much improved cycle performance and rate capability than the low dense structure (LDGS). We also evaluated the volumetric capacity of the high-density graphene/sulfur hybrid in Fig. 3d. Although such a hybrid does not have very high sulfur fraction, it still shows comparative volumetric performance. Further efforts are ongoing to increase the sulfur fraction to a satisfactory level for practical applications. This strategy avoids the insulting problem with high sulfur fraction and the compact structure can further accelerate the electron transfer among the interlinked graphene sheets. Our approach provides an effective solution for the advanced cathode with high volumetric performance in Li-S batteries and more efforts are being made to combine densification of the carbon matrix and increasing sulfur content to obtain a Li-S battery with higher volumetric performance.

The volume expansion of sulfur, which is another crucial issue for Li-S batteries, is in contradiction with the volumetric performance since some void space should be left in carbon for accommodating the volume variation, further decreasing the material density and volumetric performance. On the other hand, less void space/pore volume of carbon material can enhance the confinement of polysulfides during recharging. Therefore, pore structure optimization is required to guarantee the buffering of sulfur as well as enough confinement effect of polysulfides. 3D graphene/sulfur assembly actually provides us a good example, whose pore structure can be well tuned by altering or combining different drying strategies to optimize the pore structure. The "Shumai" structure we mentioned above has some elastic features if the pore structure can be precisely controlled [11]. Further study is ongoing to tailor the evolution of pore structure of 3D graphene/sulfur assembly in the drying process, and to propose an optimized pore structure which leads to suitable density, ion transport channels and void space for accommodating volume change.

In summary, great advances have been achieved recently towards Li-S batteries with superior volumetric performance. But it is still challenging in the near future to develop high volumetric performance carbon/sulfur cathode 
a

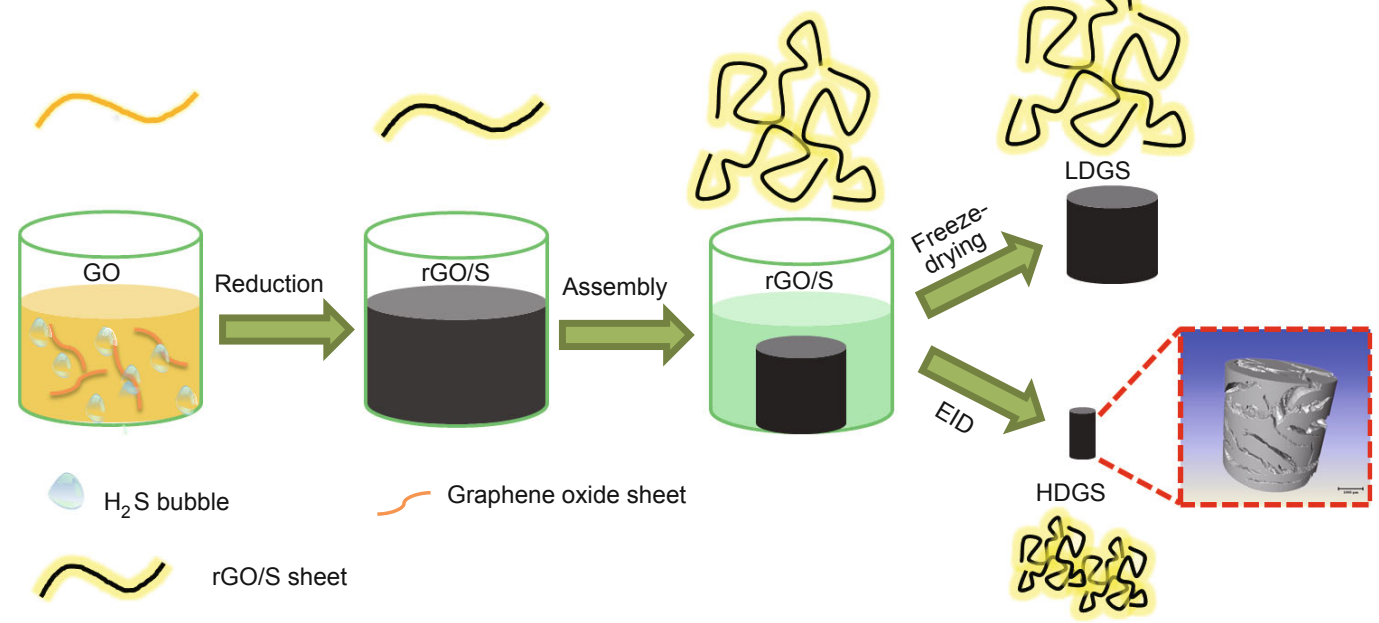

b

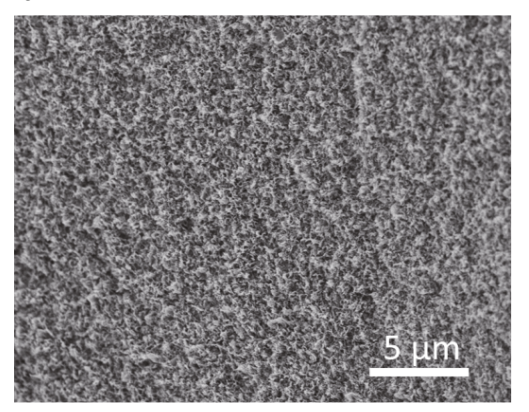

C

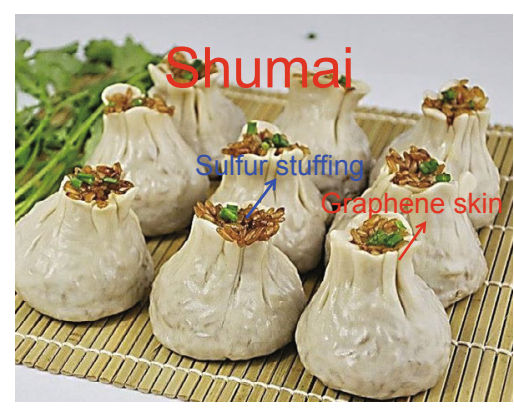

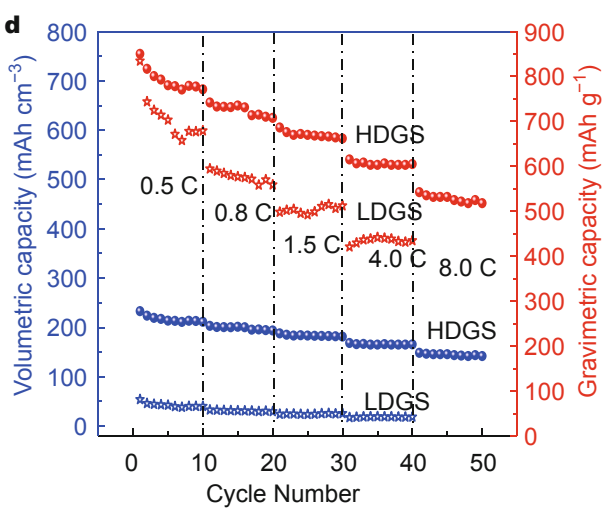

Figure 3 (a) Schematic of the formation of HDGS and LDGS. (b) SEM image of the HDGS. (c) Illustration of the formation of "Shumai" structure composed of a graphene skin and sulfur stuffing. (d) Rate performance of HDGS and LDGS. Adapted with permission from Ref. [25], Copyright 2015, Royal Society of Chemistry.

with high structural stability, low shuttle effect and low cost. The pace towards the high volumetric performance Li-S batteries must be accelerated to meet the increasing demand, since volumetric performance of EES devices is critical for upgrading the electricity-driven systems. Although it is still in its infancy to investigate volumetric performance for Li-S batteries, several pioneered studies have demonstrated the potential to achieve high volumetric performance by materials design and structure control. High sulfur content and robust carbon matrix are promising remedies for this issue. Meanwhile, we also need to make efforts on designing advanced anode materials, seeking more stable electrolyte and suitable separator. Solid and flexible Li-S batteries are also important directions for their future development. It is hopefully that the integration of materials science, electrochemical process optimization and industrial design will make breakthroughs for high volumetric performance Li-S batteries, further extending to other EES devices.
Received 30 April 2015; accepted 8 May 2015; published online 13 May 2015

1 Zhang C, Lv W, Tao Y, Yang QH. Towards superior volumetric performance: design and preparation of novel carbon materials for energy storage. Energy Environ Sci, 2015, 9: 1390-1403

2 Bruce PG, Freunberger SA, Hardwick LJ, Tarascon JM. $\mathrm{Li}_{-} \mathrm{O}_{2}$ and Li-S batteries with high energy storage. Nat Mater, 2012, 11: 19-29

3 Wang DW, Zeng QC, Zhou GM, et al. Carbon-sulfur composites for Li-S batteries: status and prospects. J Mater Chem A, 2013, 1: 9382-9394

4 Zhou GM, Li F, Cheng HM. Progress in flexible lithium batteries and future prospects. Energy Environ Sci, 2014, 7: 1307-1338

5 Zhang Q, Cheng XB, Huang JQ, et al. Review of carbon materials for advanced lithium-sulfur batteries. New Carbon Mater, 2014, 29: 241-264

6 Gogotsi Y, Simon P. True performance metrics in electrochemical energy storage. Science, 2011, 334: 917-918

7 Simon P, Gogotsi Y. Capacitive energy storage in nanostructured carbon-electrolyte systems. Acc Chem Res, 2013, 46: 1094-1103

8 Tao Y, Xie XY, Lv W, et al. Towards ultrahigh volumetric capacitance: graphene derived highly dense but porous carbons for supercapacitors. Sci Rep, 2013, 3: 2975 
9 Yang Y, Zheng GY, Cui Y. Nanostructured sulfur cathodes. Chem Soc Rev, 2013, 42: 3018-3032

10 Tao Y, Kong DB, Zhang C, et al. Monolithic carbons with spheroidal and hierarchical pores produced by the linkage of functionalized graphene sheets. Carbon, 2014, 69: 169-177

11 Qiu L, Liu JZ, Chang SLY, et al. Biomimetic superelastic graphenebased cellular monoliths. Nat Commun, 2012, 3: 1241

12 Yang XW, Cheng C, Wang YF, et al. Liquid-mediated dense integration of graphene materials for compact capacitive energy storage. Science, 2013, 341: 534-537

13 Cheng XB, Huang JQ, Zhang Q, et al. Aligned carbon nanotube/sulfur composite cathodes with high sulfur content for lithium-sulfur batteries. Nano Energy, 2014, 4: 65-72

14 Peng HJ, Liang JY, Zhu L, et al. Catalytic self-limited assembly at hard templates: a mesoscale approach to graphene nanoshells for lithium-sulfur batteries. ACS Nano, 2014, 8: 11280-11289

15 Zhu L, Zhu WC, Cheng XB, et al. Cathode materials based on carbon nanotubes for high-energy-density lithium-sulfur batteries. Carbon, 2014, 75: 161-168

16 Zhao MQ, Peng HJ, Tian GL, et al. Hierarchical vine-tree-like carbon nanotube architectures: in-situ CVD self-assembly and their use as robust scaffolds for lithium-sulfur batteries. Adv Mater, 2014, 26: 7051-7508

17 Tang C, Zhang Q, Zhao MQ, et al. Nitrogen-doped aligned carbon nanotube/graphene sandwiches: facile catalytic growth on bifunctional natural catalysts and their applications as scaffolds for highrate lithium-sulfur batteries. Adv Mater, 2014, 26: 6100-6105

18 Peng HJ, Huang JQ, Zhao MQ, et al. Nanoarchitectured graphene/ CNT@porous carbon with extraordinary electrical conductivity and interconnected micro/mesopores for lithium-sulfur batteries. Adv Funct Mater, 2014, 24: 2772-2781

19 Zhao MQ, Zhang Q, Huang JQ, et al. Unstacked double-layer templated graphene for high-rate lithium-sulphur batteries. Nat Commun, 2014, 5: 3410

20 Cheng XB, Peng HJ, Huang JQ, et al. Three-dimensional aluminum foam/carbon nanotube scaffolds as long- and short-range electron pathways with improved sulfur loading for high energy density lithium sulfur batteries. J Power Sources, 2014, 261: 264-270

21 Zhou GM, Li L, Ma CQ, et al. A graphene foam electrode with high sulfur loading for flexible and high energy Li-S batteries. Nano Energy, 2015, 11: 356-365

22 Zhou GM, Yin LC, Wang DW, et al. Fibrous hybrid of graphene and sulfur nanocrystals for high-performance lithium-sulfur batteries. ACS Nano, 2013, 7: 5367-5375

23 Lv W, Li ZJ, Zhou GM, et al. Tailoring microstructure of graphenebased membrane by controlled removal of trapped water inspired by the phase diagram. Adv Funct Mater, 2014, 24: 3456-3463

24 Li H, Yang X, Wang X, et al. Dense integration of graphene and sulfur through the soft approach for compact lithium/sulfur battery cathode. Nano Energy, 2015, 12: 468- 475

25 Zhang C, Liu DH, Lv W, et al. A high-density graphene-sulfur assembly: a promising cathode for compact Li-S batteries. Nanoscale, 2015, 7: 5592-5597

26 Zhang C, Lv W, Zhang WG, et al. Reduction of graphene oxide by hydrogen sulfide: a promising strategy for pollutant control and as an electrode for Li-S batteries. Adv Energy Mater, 2014, 4: 1301565

27 Chen WF, Yan LF. In situ self-assembly of mild chemical reduction graphene for three-dimensional architectures. Nanoscale, 2011, 3: 3132-3137

28 Lv W, Tao Y, Ni W, et al. One-pot self-assembly of three-dimensional graphene macroassemblies with porous core and layered shell. J Mater Chem, 2011, 21: 12352-12357

29 Lv W, Zhang C, Li Z, Yang QH. Self-assembled 3D graphene monolith from solution. J Phys Chem Lett, 2015, 6: 658-668

Acknowledgements This work was supported by the National Basic Research Program of China (2014CB932400), National Natural Science Foundation of China (U1401243, 51372167 and 51311140260) and Shenzhen Basic Research Project (ZDSYS20140509172959981). We also thank Prof. Qiang Zhang at Tsinghua University for his comments and suggestions.

Author contributions Yang QH conceived the structure of this contribution and Zhang $\mathrm{C}$ was mainly responsible for preparing the initial manuscript.

Conflict of interest The authors declare that they have no conflict of interest. 


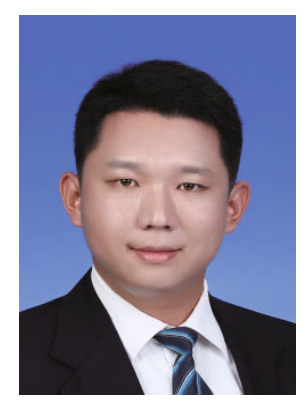

Chen Zhang received his BSc degree from Tianjin University in 2010 and continued his study as a PhD candidate under the supervision of Prof. Quan-Hong Yang till now. His research interest includes the design and controlled preparation of graphene-based materials for compact energy storage devices.

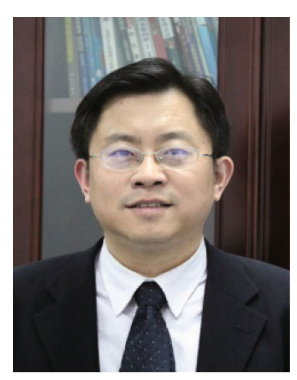

Quan-Hong Yang was born in 1972 and joined Tianjin University as a full professor of nanomaterials in 2006. He is now also leading a graphene lab as co-director at the Graduate School at Shenzhen, Tsinghua University. His research has been totally focused on novel carbon materials and their applications since the middle of 1990s. His recent research interests include mainly two related aspects: interfacial assembly of graphene for production of functional carbons and carbon-based energy storage devices with high volumetric energy density. See http://nanoyang.tju.edu.cn for more details.

中文摘要 随着人们对于储能器件微型化、长寿命需求的日益增加, 电化学储能器件的体积能量密度成为其重要性能指标. 锂硫电池 作为下一代重点发展的高比能量二次电池, 其先进电极材料的研发受到越来越多的关注. 碳材料的使用可有效提高器件性能, 然而较 低的电极密度导致锂硫电池体积能量密度较低. 通过对电极材料的设计以及结构调控, 实现电极材料致密化, 可以有效解决这一问题. 一方面, 提高复合电极材料中硫的含量, 可以提高材料整体密度, 减少非活性组分, 提升电极体积比容量; 另一方面, 通过实现碳材料的 致密化, 也可以提高碳/硫复合电极材料密度的调控, 获得具有高体积比容量的电极材料. 本文对目前关于高体积比容量锂硫电池电极 材料的研究工作进行了评述, 虽然目前这一方向的研究尚处起步阶段, 但其必将成为未来锂硫电池研究的重点. 通过集成材料设计、 组装、器件结构优化等多方面工作, 能够实现高体积比容量电极材料和高体积能量密度锂硫电池的应用, 推动电化学储能领域的发展. 